\title{
Association between cannabinoid 1 receptor availability and glutamate levels in healthy controls and drug-free patients with first episode psychosis: a multi-modal PET and 1H-MRS study
}

\author{
Faith Borgan ${ }^{1,2}$ (D) Mattia Veronese ${ }^{3} \cdot$ Tiago Reis Marques $^{1,2} \cdot$ David J. Lythgoe $^{3} \cdot$ Oliver Howes $^{1,2}$
}

Received: 24 March 2020 / Accepted: 20 August 2020 / Published online: 28 September 2020

(c) The Author(s) 2020

\begin{abstract}
Cannabinoid 1 receptor and glutamatergic dysfunction have both been implicated in the pathophysiology of schizophrenia. However, it remains unclear if cannabinoid 1 receptor alterations shown in drug-naïve/free patients with first episode psychosis may be linked to glutamatergic alterations in the illness. We aimed to investigate glutamate levels and cannabinoid 1 receptor levels in the same region in patients with first episode psychosis. Forty volunteers ( 20 healthy volunteers, 20 drugnaïve/free patients with first episode psychosis diagnosed with schizophrenia/schizoaffective disorder) were included in the study. Glutamate levels were measured using proton magnetic resonance spectroscopy. CB1R availability was indexed using the distribution volume $\left(\mathrm{V}_{\mathrm{T}}\left(\mathrm{ml} / \mathrm{cm}^{3}\right)\right)$ of $\left[{ }^{11} \mathrm{C}\right] \mathrm{MePPEP}$ using arterial blood sampling. There were no significant associations between ACC CB1R levels and ACC glutamate levels in controls $(R=-0.24, p=0.32)$ or patients $(R=-0.10, p=0.25)$. However, ACC glutamate levels were negatively associated with CB1R availability in the striatum $(R=-0.50, p=0.02)$ and hippocampus $(R=-0.50, p=0.042)$ in controls, but these associations were not observed in patients $(p>0.05)$. Our findings extend our previous work in an overlapping sample to show, for the first time as far as we're aware, that cannabinoid 1 receptor alterations in the anterior cingulate cortex are shown in the absence of glutamatergic dysfunction in the same region, and indicate potential interactions between glutamatergic signalling in the anterior cingulate cortex and the endocannabinoid system in the striatum and hippocampus.
\end{abstract}

Keywords Glutamate $\cdot$ Cannabinoid $\cdot$ Endocannabinoid system $\cdot$ Cannabinoid 1 receptor $\cdot$ Schizophrenia

Electronic supplementary material The online version of this article (https://doi.org/10.1007/s00406-020-01191-2) contains supplementary material, which is available to authorized users.

Faith Borgan

faith.borgan@kcl.ac.uk

1 Psychosis Studies Department, Institute of Psychiatry, Psychology and Neuroscience, King's College London, London, England

2 Institute of Clinical Sciences, Faculty of Medicine, Imperial College London, Hammersmith Hospital, London W12 0NN, UK

3 Centre for Neuroimaging Sciences, Institute of Psychiatry, Psychology and Neuroscience, King's College London, London, England

\section{Introduction}

Schizophrenia is ranked as one of the most disabling health conditions [1], with annual costs ranging between 94 million to 102 billion dollars across a range of countries [2]. While current pharmacological treatments predominately block the D2 dopamine receptor [3], they have limited efficacy in reducing symptom severity in $30 \%$ of patients $[4,5]$ and they fail to improve cognitive deficits $[6,7]$. In view of these limitations, further work is needed to identify therapeutic mechanisms and biomarkers that may be useful for targeting both psychotic and cognitive symptoms in schizophrenia [8-10].

The pathophysiology of schizophrenia has been postulated to involve glutamatergic dysfunction [11, 12], since $\mathrm{N}$-methyl-D-aspartate receptor (NMDAR) antagonists induce positive and negative symptoms and cognitive impairments in healthy controls, mimicking the phenotype of schizophrenia [13-15]. In particular, low doses of 
ketamine, an NMDAR antagonist, induce psychotic symptoms, cognitive impairments, and increase anterior cingulate cortex (ACC) glutamate levels in healthy volunteers [16]. While this finding was taken to suggest that altered ACC glutamate levels may be associated with the induction of psychotic symptoms, other studies have shown that ketamine induces psychotic symptoms in the absence of any change in ACC glutamate levels [17, 18].

Glutamate levels can be measured in vivo using proton magnetic resonance spectroscopy (1H-MRS). Discrepant findings have been reported in chronic patients taking antipsychotic medication including reports of no differences [19] and decreased glutamate levels in the anterior cingulate cortex (ACC) [20, 21]. In contrast, patients with first episode psychosis who are not taking antipsychotics have largely been shown to have no differences in ACC glutamate levels relative to healthy volunteers [22-25], with the exception of one study reporting decreased ACC glutamate levels [26]. Despite the absence of group differences in ACC glutamate levels in drug-free patients with first episode psychosis, ACC glutamate levels have been shown to be negatively associated with symptom severity and striatal dopamine synthesis capacity [27].

Excitatory and inhibitory synaptic transmission is regulated by the cannabinoid 1 receptor $\left(\mathrm{CB}_{1} \mathrm{R}\right)$, a G-proteincoupled receptor distributed on pre-synaptic nerve terminals of glutamate and GABA interneurons throughout the cortex, hippocampus, and striatum [28]. $\mathrm{CB}_{1} \mathrm{R}$ activation transiently suppresses neurotransmitter release by the inhibition of N-, P-, and Q-type calcium channel openings and through activation of inwardly rectifying potassium channels on the pre-synaptic neuron [29-31] and adjacent neurons containing $\mathrm{CB}_{1} \mathrm{R}$ within a within $<20 \mu \mathrm{m}$ radius [29, 32]. However, these effects can be blocked by $\mathrm{CB}_{1} \mathrm{R}$ antagonists or the genetic ablation of the $\mathrm{CB}_{1} \mathrm{R}$ [33].

Since $\mathrm{CB}_{1} \mathrm{R}$ activation inhibits synaptic transmission at both glutamate and GABA neurons in the ACC, $\mathrm{CB}_{1} \mathrm{R}$ activation regulates both excitatory and inhibitory synaptic transmission. In particular, $\mathrm{CB}_{1} \mathrm{R}$ activation at GABAergic interneurons in the ACC disinhibits downstream synaptic transmission [34], whereas $\mathrm{CB}_{1} \mathrm{R}$ activation on glutamate neurons in the ACC inhibits glutamate release [35]. These findings indicate that $\mathrm{ACC} \mathrm{CB}_{1} \mathrm{R}$ regulate both excitatory and inhibitory synaptic transmission.

Previous literature has shown that $\mathrm{CB}_{1} \mathrm{R}$ agonists modulate glutamate release in rodents [36, 37] and humans [38]. Moreover, mice with $\mathrm{CB}_{1} \mathrm{R}$ deletions on cortical glutamate neurons exhibit enhanced excitability on cortical glutamate neurons leading to stronger seizures following the kainic acid induced excitation in hippocampal pyramidal neurons as well as the significant loss of glutamatergic neurons $[39,40]$.
Since we recently showed that drug-free patients with schizophrenia have lower levels of $\mathrm{CB}_{1} \mathrm{R}$ in the $\mathrm{ACC}$, and that greater reductions were linked to greater cognitive deficits [41], we specifically aimed to investigate whether $\mathrm{CB}_{1} \mathrm{R}$ alterations in the ACC may be associated with glutamatergic alterations in the $\mathrm{ACC}$ in the same drug-free patients. We hypothesized that $A C C \mathrm{CB}_{1} \mathrm{R}$ levels would be associated with ACC glutamate levels. We also aimed to conduct exploratory analyses to investigate the association between ACC glutamate levels and CB1 receptors in other brain regions where $\mathrm{CB}_{1} \mathrm{R}$ levels are altered in schizophrenia [41-43]. We also aimed to investigate the association between ACC glutamate levels, symptom severity, and cognitive functioning, as determined by the Wechsler Digit Symbol Coding test [44] based on evidence that it is highly corelated with total cognitive deficits in first episode psychosis [45].

\section{Methods}

\section{Ethics statement}

Relevant ethical approvals were obtained from the Camberwell St. Giles Research Ethics Committee (14/LO/1289). Informed written consent was obtained from all subjects prior to participation.

\section{Participants}

A total of 40 volunteers ( 20 healthy volunteers and 20 drugfree patients with first episode psychosis) were recruited. All patients were recruited from early intervention services for psychosis in London, United Kingdom. Age (age \pm 3 years) and sex-matched healthy volunteers were recruited via local advertising.

\section{Inclusion/exclusion criteria}

First episode psychosis patients met the following inclusion criteria: (1) diagnosis of a psychotic disorder as determined by the ICD-10; (2) antipsychotic naïve or antipsychotic free for at least 6 months, as determined by medical records and self-report; (3) no current use of any concurrent pharmacological treatments as determined by medical records and self-report (e.g., benzodiazepines, antidepressants etc.); (4) illness duration of less than 3 years as determined by medical records and self-report.

Healthy volunteers met the additional inclusion criteria including: (1) no current or lifetime history of an Axis-I disorder as determined by the Structured Clinical Interview for DSM-IV-TR (SCID-I/P) [46] and (2) no family history of an Axis-I disorder in first- and second-degree relatives 
as determined by the Family Inventory for Genetics studies (FIGS) [47]. Exclusion criteria for all volunteers: (1) reported current use of any recreational substances within the last month; (2) screened positive on a urine toxicology test that can detect THC metabolite THCCOOH for up to 30 days (50 ng/ml cut off) (SureScreen, Diagnostics; $50 \mathrm{ng} /$ $\mathrm{ml}$ cut off); (3) screened positive on a multi-panel drug screen detecting the following substances: amphetamine (300 ng/ml cut off), cocaine (150 ng/ml cut off), ketamine $(1000 \mathrm{ng} / \mathrm{ml}$ cut off), methamphetamine (300 ng/ml cut off), and opiates (2000 ng/ml cut off) (SureScreen Diagnostics).

Volunteers were aged $18-35$ and were able to give informed, written consent. Volunteers were excluded if they had: (1) a history of a head injury leading to loss of consciousness, (2) personal or family history of neurological or physical health problems, (3) contraindications to MRI safety including head trauma, pregnancy, the presence of metal plates, pins, bridges, or dentures; or 4) current history of substance use or dependence as determined by the Structured Clinical Interview for DSM-IV-TR (SCID-I/P) [46].

\section{Demographics}

Age, sex, ethnicity, and years of education were recorded.

\section{Clinical measures}

Clinical symptom severity was determined by trained staff using the Positive and Negative Syndrome scale (PANSS) [48]. Social and occupational functioning was measured using the Global Assessment of Functioning (GAF) [49]. Healthy volunteers were screened for personal and family history of mental health problems using SCID-I/P [46] and FIGS [47]. Cognition was measured using the Wechsler Digit Symbol Coding test [44] based on evidence that it is highly corelated with total cognitive deficits in first episode psychosis [45].

\section{Neuroimaging}

\section{Positron emission tomography}

CB1R-selective radiotracer, $\left[{ }^{11} \mathrm{C}\right] \mathrm{MePPEP}$, was used to investigate the distribution volume of the cannabinoid 1 receptor. A continuous 90-min PET scan acquired using a Hi-Rez Biograph 6 CT44931 scanner in three-dimensional mode, following a bolus injection $($ mean $=314$; $\mathrm{SD}=34.4 \mathrm{MBq})$ of $\left[{ }^{11} \mathrm{C}\right] \mathrm{MePPEP}$ synthesized using methods reported elsewhere [50,51]. CT scans were acquired prior to each PET scan for correction for attenuation and scatter. Continuous arterial blood sampling took place for the first $15 \mathrm{~min}$ of the scan which was followed by discrete blood sampling at $2,5,10,15,20,25,35,40,50,60$,
70,80 , and 90 min after the radioligand injection. Images were reconstructed with filtered back projection including corrections for attenuation and scatter. PET scans were performed between 9:00 AM and 3 PM; participants fasted (water was allowed) and abstained from alcohol and substances for more than $12 \mathrm{~h}$ before undergoing the scan. PET and MRI scans were conducted approximately 2 weeks apart.

\section{Magnetic resonance imaging and proton magnetic resonance spectroscopy (1H-MRS)}

MRI scans were acquired using the General Electric MR750 3.0 T MRI scanner. High-resolution 3D T1-weighted structural MRI images were acquired for the 1H-MRS voxel prescription and anatomical co-localisation (in-plane matrix size of $256 \times 256, \mathrm{FOV}=26.0 \mathrm{~mm}$ ) using a whole-brain, sagittal IR-SPGR acquisition, and an eight-channel head coil $(\mathrm{TR}=7.34 \mathrm{~mm}, \mathrm{TI}=400 \mathrm{~ms}$, inversion time $=4 \mathrm{~s}$, flip angle $=11^{\circ}$, and slice thickness $=1.2 \mathrm{~mm}$ ). Data included here were previously published as a part of a multi-centre study [41]. The neuroimaging acquisition parameters for the acquisition of the proton magnetic resonance spectroscopy data were as follows: internal localizer scans were used to determine the anterior commissure-posterior commissure line and inter-hemispheric angle. For the voxel placement, the sagittal IR-SPGR scans were reformatted into axial and coronal planes and the voxel positioned in the ACC. This was followed by auto pre-scans for optimisation of water suppression and shimming of the MRS voxel. $1 \mathrm{H}-\mathrm{MRS}$ spectra were acquired from the anterior cingulate region-of-interest (right-left $20 \mathrm{~mm} \times$ anterior-posterior $20 \mathrm{~mm} \times$ superior-inferior $20 \mathrm{~mm}$ ). The placement of the anterior cingulate voxel was based on the midline sagittal localizer with the centre of the $20 \mathrm{~mm} \times 20 \mathrm{~mm} \times 20 \mathrm{~mm}$ voxel placed $13 \mathrm{~mm}$ above the anterior portion of the genu of the corpus callosum, perpendicular to the anterior commissure-posterior commissure line to minimize the inclusion of white matter and cerebral spinal fluid (CSF) (see Fig. 1 for images of the coronal, axial, and sagittal placement of the voxel). Finally, the ${ }^{1} \mathrm{H}-\mathrm{MRS}$ spectra (Point RESolves Spectroscopy (PRESS), TE $=30 \mathrm{~ms}, \mathrm{TR}=2 \mathrm{~s}$ ) were obtained through the PROton Brain Examination (PROBE) sequence by GE, which includes water suppression. Water unsuppressed scans were also acquired for subsequent eddy current correction and water referenced metabolite quantification. A subset of these data (17 subjects) were reported previously [25]. As described previously [52], MRI scans were performed between 9:00 and 11:30 AM; participants fasted (water was allowed) and abstained from alcohol and other substances for more than $12 \mathrm{~h}$ before undergoing the MRI scan. 

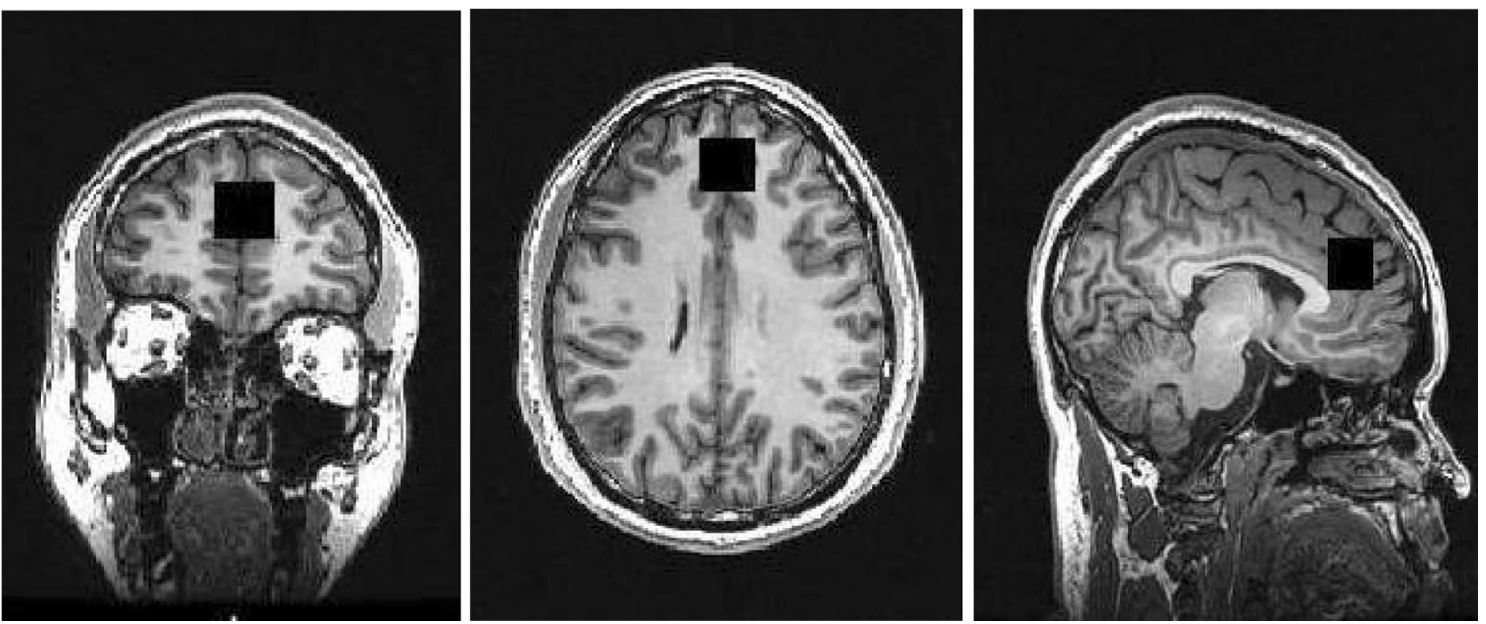

Fig. 1 Coronal (left), axial (middle), and sagittal (right) planes depicting the placement of the voxel over the anterior cingulate during the proton magnetic resonance spectroscopy scan. The placement of the anterior cingulate voxel was based on the midline sagittal localizer with the centre of the $20 \mathrm{~mm} \times 20 \mathrm{~mm} \times 20 \mathrm{~mm}$ voxel placed $13 \mathrm{~mm}$ above the anterior portion of the genu of the corpus callosum, perpendicular to the anterior commissure-posterior commissure line to minimize the inclusion of white matter and cerebral spinal fluid

\section{Analysis}

\section{Imaging analysis}

\section{Positron emission tomography}

Methods were reported previously [41]. Data pre-processing was performed using a combination of Statistical Parametric Mapping 12 (https://www.fil.ion.ucl.ac.uk/spm) and FSL (https://www.fsl.fmrib.ox.ac.uk/fsl) functions, as implemented in MIAKAT Version 1.0 (miakat.org) using MATLAB R2015b [53]. Motion correction was applied to non-attenuation corrected images [54]. Non-attenuatedcorrected frames were realigned to a single "reference" frame by employing a mutual information algorithm. The transformation parameters were then applied to the corresponding attenuated-corrected dynamic images, creating a movement-corrected dynamic image which was used for the analysis. Realigned frames were then summated to create single-subject motion-corrected maps which were then used for MRI and PET co-registration prior to PET data quantification. T1-weighted structural images were co-registered to the PET image using rigid body transformation. Normalization parameters were obtained by warping the co-registered structural MRI to MNI space (International Consortium for Brain Mapping ICBM/MNI) using bias-corrected segmentation. The inverse of these parameters was used to fit a neuroanatomical atlas to each individual PET scan using the Hammersmith atlas [55]. Whole blood time-activity curves (TACs) were fitted using a multi-exponential function as derived by Feng's model [56]. For each scan, a time delay was fitted and applied to the input functions (both parent and whole blood TACs) to account for any temporal delay between blood sample measurement and target the tissue data. Voxel-based morphometry analyses reported elsewhere [41] indicated that there were no significant volumetric differences between patients and controls in the anterior cingulate cortex, and thus, no partial volume corrections were conducted.

$\mathrm{CB}_{1} \mathrm{R}$ distribution volume $\left(\mathrm{V}_{\mathrm{T}}\right)$ was calculated using the Logan graphical method with a metabolite-free arterial plasma input function [57] using a regional approach. An independently derived region of interest of the anterior cingulate cortex was obtained from a standard probabilistic atlas [55]. A 3D image containing the MNI coordinates used for the MRS voxel placement was also generated using SPM12, so that ACC $V_{T}$ values could be extracted from the ACC MRS voxel using MATLAB R2015b [53]. The ACC was selected given prior findings implicating this region in the pathophysiology of psychotic and cognitive symptoms $[58,59]$, and findings indicated that the $\mathrm{CB}_{1} \mathrm{R}$ regulates synaptic transmission in this region $[29,60]$.

\section{Proton magnetic resonance spectroscopy}

Methods were reported previously [25]. Water-scaled metabolites were analysed using LC-model 6.3-0I using an experimentally acquired basis set to estimate concentrations of 16 metabolites including glutamate, glutamine, GLX (glutamate + glutamine), N-Acetyl-Aspartate (NAA), L-alanine, aspartate, creatine, phosphocreatine, GABA, glucose, glycerophosphocholine, glycine, myo-inositol, L-lactate, $\mathrm{N}$-acetylaspartylglutamate, phosphocholine, and taurine. Metabolite analyses were restricted to spectra with 
linewidth (full-width at half-maximum; FWHM) $\leq 0.1 \mathrm{ppm}$, Cramér-Rao lower bounds (CRLB) for glutamate $\leq 20 \%$, and signal-to-noise ratio $\geq 5$. Corrections were applied to take into account the relative distribution of cerebrospinal fluid within the anterior cingulate. In-house scripts written in Python were used to identify the relative distribution of white matter, grey matter, and cerebrospinal fluid in the 8 $\mathrm{cm}^{3}$ voxel prescribed to the anterior cingulate. The following correction described previously [61] was subsequently applied to correct for CSF, grey matter (GM), and white matter (WM) content within the $8 \mathrm{~cm}^{3}$ voxel, where $\mathrm{M}=$ raw metabolite value:

$\operatorname{Mcorr}=M\left(\frac{\mathrm{WM}+(\mathrm{GM} \times 1.22)+(\mathrm{CSF} \times 1.55)}{(\mathrm{WM}+\mathrm{GM})}\right)$.

Apart from assuming the $T_{2}=80 \mathrm{~ms}$ for tissue water, no corrections were applied for tissue and metabolite $\mathrm{T}_{1}$ and
$\mathrm{T}_{2}$ relaxation. The equation also includes a correction for the default assumption the voxel is pure WM during the LCModel analysis Table 1.

\section{Statistical analysis}

The Statistical Package for the Social Sciences (version 22) [62] was used for all statistical analyses. Data normality was assessed using the Shapiro-Wilk test.

Independent samples $t$ tests were also used to investigate group differences in experimental PET variables that may potentially confound the results including weight, dose $(\mathrm{Mbq})$, injected mass $(\mu \mathrm{g})$, specific activity $(\mathrm{GBq} / \mu \mathrm{mol})$, fraction of ligand free in plasma (fp), and total motion (frame-to-frame displacement) (see Table 2) and experimental $1 \mathrm{H}-\mathrm{MRS}$ variables that may potentially confound the results including linewidth, signal-to-noise, and data shift (see Table 3).

Table 1 Demographics

\begin{tabular}{|c|c|c|}
\hline & Healthy volunteers & First episode psychosis patients \\
\hline$N$ & 20 & 20 \\
\hline Age (years) mean $(\mathrm{SD})$ & Mean $=27.15 ; \mathrm{SD}=6.12$ & $\begin{array}{l}\text { Mean }=27.00 \\
\mathrm{SD}=5.06\end{array}$ \\
\hline Sex (male/female) & $20 / 0$ & $20 / 0$ \\
\hline Ethnicity (CAUCASIAN/black African or Caribbean/Asian/mixed) & $7 / 2 / 9 / 2$ & $10 / 4 / 5 / 1$ \\
\hline Diagnosis (schizophrenia/schizoaffective disorder) & N/A & $18 / 2$ \\
\hline Illness duration (months) mean (SD) & N/A & $\begin{array}{l}\text { Mean }=22.66 \\
S D=11.64\end{array}$ \\
\hline Duration of prior treatment (if applicable) (months) mean (SD) & N/A & $\begin{array}{l}\text { Mean }=4.21 \\
\mathrm{SD}=5.44\end{array}$ \\
\hline $\begin{array}{l}\text { Current use of antipsychotics } \\
\text { (yes/no) }\end{array}$ & N/A & $0 / 20$ \\
\hline Prior use of antidepressant (yes/no) & N/A & $5 / 15$ \\
\hline Prior use of antipsychotics (yes/no) & N/A & $16 / 4$ \\
\hline $\begin{array}{l}\text { PANSS positive } \\
\text { mean (SD) }\end{array}$ & N/A & $\begin{array}{l}\text { Mean }=26.95 \\
S D=17.75\end{array}$ \\
\hline $\begin{array}{l}\text { PANSS negative } \\
\text { mean (SD) }\end{array}$ & N/A & $\begin{array}{l}\text { Mean }=22.79 \\
\mathrm{SD}=6.96\end{array}$ \\
\hline $\begin{array}{l}\text { PANSS general } \\
\text { mean }(\mathrm{SD})\end{array}$ & N/A & $\begin{array}{l}\text { Mean }=39.74 \\
S D=10.77\end{array}$ \\
\hline $\begin{array}{l}\text { PANSS total } \\
\text { mean }(\mathrm{SD})\end{array}$ & N/A & $\begin{array}{l}\text { Mean }=84.21 \\
\mathrm{SD}=22.10\end{array}$ \\
\hline PANSS five-factor positive mean (SD) & N/A & mean $=14.61 \mathrm{SD}=4.34$ \\
\hline PANSS five-factor negative mean (SD) & N/A & Mean $=24.94 \mathrm{SD}=7.28$ \\
\hline PANSS factor cognitive disorganization mean (SD) & N/A & Mean $=18.61 \mathrm{SD}=6.17$ \\
\hline PANSS five-factor depression/anxiety mean (SD) & N/A & Mean $=8.50 \mathrm{SD}=2.94$ \\
\hline PANSS five-factor excitability/hostility mean (SD) & N/A & Mean $=6.28 \mathrm{SD}=3.51$ \\
\hline Current cannabis use (yes/no) & $0 / 20$ & $0 / 20$ \\
\hline Current alcohol use (yes/no/missing data) & $12 / 8 / 0$ & $12 / 8 / 2$ \\
\hline
\end{tabular}

$N$ sample size, FEP first episode psychosis, $t$ independent samples $t$ test, $\chi^{2}$ Chi-square, $d f$ degrees of freedom, $N / A$ not applicable, $S D$ standard deviation, $B P R S$ brief psychiatric rating scale

All demographics reported in this table were reported previously [41] 
Table 2 Experimental PET variables

\begin{tabular}{|c|c|c|c|c|c|}
\hline & Healthy volunteers & FEP patients & $\mathrm{T}$ & $\mathrm{df}$ & $p$ \\
\hline$N$ & 20 & 20 & & & \\
\hline Weight $(\mathrm{kg})$ & $M=78.29 ; \mathrm{SD}=13.26$ & $M=85.09 ; \mathrm{SD}=14.17$ & -1.568 & 38 & 0.13 \\
\hline Body mass index ${ }^{a}$ & $M=25.47 ; \mathrm{SD}=3.78$ & $M=26.65 ; \mathrm{SD}=5.24$ & -0.674 & 26 & 0.51 \\
\hline Dose $(\mathrm{MBq})$ & $M=311.32 ; \mathrm{SD}=44.87$ & $M=311.50 ; \mathrm{SD}=27.48$ & 0.016 & 38 & 0.98 \\
\hline Injected mass $(\mu \mathrm{g})$ & $M=4.31 ; \mathrm{SD}=1.60$ & $M=4.72 . \mathrm{SD}=2.46$ & -0.583 & 38 & 0.56 \\
\hline Specific activity $\mathrm{GBq} / \mu \mathrm{mol}$ & $M=97.32 ; \mathrm{SD}=287.91$ & $M=158.38 ; \mathrm{SD}=556.02$ & -0.436 & 38 & 0.67 \\
\hline $\mathrm{Fp}(\%$ if $>1$ or fraction if $<1)$ & $M=0.19 ; \mathrm{SD}=4.68$ & $M=0.16 ; \mathrm{SD}=0.05$ & 1.758 & 38 & 0.09 \\
\hline Total scanner motion $(\mathrm{mm})^{\mathrm{b}}$ & $M=12.58 ; \mathrm{SD}=4.68$ & $M=13.78 ; \mathrm{SD}=5.95$ & -0.709 & 38 & 0.48 \\
\hline
\end{tabular}

FEP first episode psychosis, $N$ number, $\mathrm{kg}$ kilograms, $m m$ millimetres, $M B q$ megabecquerel, $\mu g$ microgram, umol micromoles, $G B q$ gigabecquerel

${ }^{a}$ Body mass index, calculated using methods described previously [84]

${ }^{\mathrm{b}}$ Total scanner motion was defined as the sum of total frame-to-frame movement during imaging acquisition
Table 3 Experimental 1H-MRS variables

\begin{tabular}{llllll}
\hline & Healthy volunteers & FEP patients & $t$ & $\mathrm{df}$ & $p$ \\
\hline$N$ & 20 & 20 & & & \\
Linewidth (ppm) & $M=0.03 ; \mathrm{SD}=0.01$ & $M=0.04 ; \mathrm{SD}=0.01$ & -1.257 & 38 & 0.22 \\
Signal-to-noise & $M=26.35 ; \mathrm{SD}=6.47$ & $M=24.75 ; \mathrm{SD}=6.31$ & 0.79 & 38 & 0.43 \\
Data shift $(\mathrm{ppm})$ & $M=0.02 ; \mathrm{SD}=0.01$ & $M=0.02 ; \mathrm{SD}=0.01$ & 0.05 & 38 & 0.96 \\
\hline
\end{tabular}

ppm parts per million
To test the main hypothesis that $\mathrm{ACC} \mathrm{CB}_{1} \mathrm{R}$ availability would be associated with ACC glutamate levels, a Pearson's correlation coefficient was calculated to investigate the association between $\mathrm{ACC}$ glutamate levels and $\mathrm{ACC} \mathrm{CB}_{1} \mathrm{R}$ availability (using a standard probabilistic atlas [55] and values extracted from the whole ACC MRS voxel). Exploratory analyses were also conducted to determine whether ACC glutamate levels were associated with $\mathrm{CB}_{1} \mathrm{R}$ availability in the striatum, hippocampus, and thalamus.

Exploratory analyses were also conducted to investigate the association between ACC glutamate levels and cognition and symptom severity (PANSS total and PANSS 5-factor model described previously [63].

\section{Results}

\section{Demographics}

There were no significant differences between healthy volunteers and patients in age, gender, ethnicity, alcohol use, tobacco use, or history of cannabis abstinence in study 1 (see Table 1).

\section{Positron emission tomography: cannabinoid receptor availability}

The $\mathrm{CB}_{1} \mathrm{R}$ imaging data from this cohort have previously been reported. As we previously reported [41], patients showed significantly lower $\mathrm{CB}_{1} \mathrm{R}$ availability in the anterior cingulate cortex relative to controls (Hedge's $g=0.8$ ).

\section{Proton magnetic resonance spectroscopy (1H-MRS)}

The 1H-MRS data from this cohort have previously been reported. As we previously reported [25], there were no significant differences between healthy volunteers and patients with first episode psychosis in glutamate (see Table 4).

Table 4 1H-MRS results

\begin{tabular}{llllll}
\hline & $\begin{array}{l}\text { Healthy volun- } \\
\text { teers }\end{array}$ & $\begin{array}{l}\text { First episode psy- } t \\
\text { chosis patients }\end{array}$ & df & $p$ \\
\hline Glutamate & $15.38(1.85)$ & $16.36(1.90)$ & -1.659 & 38 & 0.11 \\
\hline
\end{tabular}




\section{Association between cannabinoid 1 receptor availability and metabolite levels}

There was no significant association between $\mathrm{ACC} \mathrm{CB}_{1} \mathrm{R}$ levels and ACC glutamate levels in controls $(R=-0.24$, $p=0.32$ ) or patients $(R=-0.10, p=0.97)$ (see Fig. 2). Since cannabinoid 1 receptor levels in the anterior cingulate cortex were extracted using a probabilistic atlas which may be different to the MRS voxel prescribed to the anterior cingulate cortex, we repeated these analyses using identical regions of interest to extract the PET and MRS data using the MRS voxel prescribed to the anterior cingulate cortex. Findings remained unchanged when investigating the association between ACC glutamate and $\mathrm{V}_{\mathrm{T}}$ values extracted from the ACC MRS voxel. In particular, there were no significant associations between ACC glutamate and ACC $\mathrm{V}_{\mathrm{T}}$ values in controls $(R=-0.055, p=0.819)$ or patients $(R=0.086$, $p=0.719)$.

Exploratory analyses in healthy volunteers indicated that ACC glutamate levels were negatively associated with $\mathrm{CB}_{1} \mathrm{R}$ availability in the striatum $(R=-0.50, p=0.02)$,

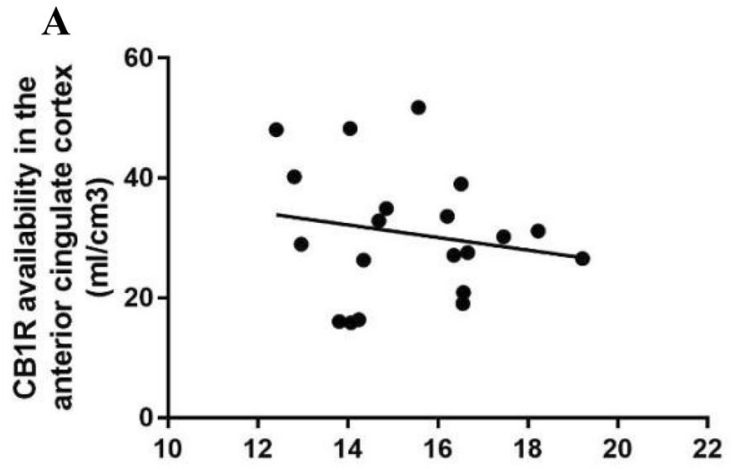

Anterior cingulate cortex glutamate levels (ppm)

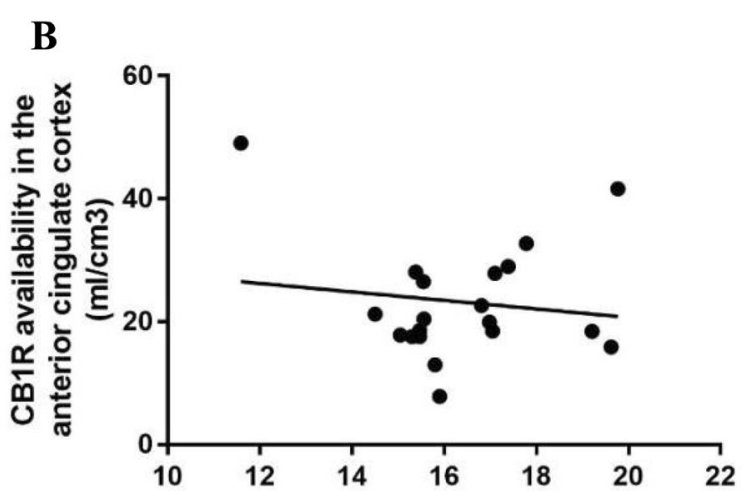

Anterior cingulate cortex glutamate levels (ppm)

Fig. 2 Scatter plot showing no association was detected between anterior cingulate cortex glutamate levels ( $\mathrm{ppm}$ ) and anterior cingulate cortex cannabinoid 1 receptor availability, indexed using the $\mathrm{V}_{\mathrm{T}}$ of $\left[{ }^{11} \mathrm{C}\right] \mathrm{MePPEP}$ in a healthy volunteers controls $(R=-0.24$, $p=0.32)$ or $\mathbf{b}$ patients $(R=-0.10, p=0.25)$ hippocampus $(R=-0.50, p=0.042)$, but not the thalamus $(R=-0.30, p=0.30)$ or the ACC $(R=-0.26, p=0.32)$. In contrast, patients failed to show any associations between ACC glutamate levels and $\mathrm{CB}_{1} \mathrm{R}$ availability in the striatum $(R=-0.30, p=0.20)$, hippocampus $(R=0.14, p=0.60)$, thalamus $(R=-0.12, p=0.61)$, or ACC $(R=-0.01$, $p=0.98)$.

\section{Association between metabolite levels and symptom severity}

There were no significant associations between total PANSS symptom severity and glutamate levels $(R=-0.10$, $p=0.69)$, GLX levels $(R=0.05, p=0.85)$, glutamine levels $(R=0.15, p=0.55)$, or NAA levels $(R=-0.03, p=0.90)$ in the ACC. These findings remained unchanged when investigating the association between PANSS subscales of positive, negative, and general symptom severity. Using a five-factor model [63], patients showed a significant positive association between glutamate levels in the anterior cingulate and the positive factor $(R=0.613, p=0.007)$ (see supplementary Figure 1), the depression/anxiety factor $(R=0.514, p=0.029)$ (see supplementary Figure 2 ) and a trend-level association with the cognitive/disorganization factor $(R=0.411, p=0.09)$.

\section{Association between metabolite levels and cognition}

There was no significant association between WAIS digit symbol coding test scores and glutamate levels in controls $(R=-0.18, p=0.48)$ or patients $(R=-0.23, p=0.34)$.

\section{Discussion}

Our findings extend our previous work in an overlapping sample showing that cannabinoid 1 receptor levels are lower in anterior cingulate cortex in drug-naïve/free patients with first episode psychosis [41] to show that these cannabinoid 1 receptor alterations are shown in the absence of evidence of glutamatergic alterations in the same brain region. In contrast to our predictions, ACC glutamate was not associated with $\mathrm{ACC} \mathrm{CB}_{1} \mathrm{R}$ availability in patients or controls. Exploratory analyses indicated that ACC glutamate levels were associated with $\mathrm{CB}_{1} \mathrm{R}$ availability in the hippocampus and striatum in controls, but not patients. We also showed that ACC glutamate levels were associated with greater symptom severity on the positive factor $(R=0.613, p=0.007)$ and the depression and anxiety factor $(R=0.514, p=0.029)$ using a five-factor model [63].This is the first study, as far as we're aware, to investigate the association between cannabinoid 1 
receptor levels and glutamate levels in humans or patients with schizophrenia.

These findings extend previous literature, showing that glutamate release can be modulated following the administration of a cannabinoid 1 receptor agonist in rodents [36, 37] and humans [38] to show that $\mathrm{CB}_{1} \mathrm{R}$ levels are related to glutamate levels in vivo in healthy individuals. These findings also extend previous work, showing that mice with CB1R deletions on cortical glutamate neurons exhibit enhanced excitability on hippocampal glutamate neurons leading to stronger seizures and neuronal loss [39, 40]. Given previous work indicating that $\mathrm{CB}_{1} \mathrm{R}$ are localised on glutamate neurons in the ACC [35], hippocampus [64], and cortical layer 5 [65], and that $\mathrm{CB}_{1} \mathrm{R}$ activation suppresses glutamate release via depolarisation-induced suppression of excitation (DSE), we expected an inverse relationship between $\mathrm{CB}_{1} \mathrm{R}$ and glutamate levels. Our finding that controls showed an association between ACC glutamate and $\mathrm{CB}_{1} \mathrm{R}$ availability in the striatum and hippocampus but not the ACC or thalamus partially support this, and highlight the need for future studies to understand endocannabinoid circuits. Our finding that patients failed to show any of the relationships between CB1R and glutamate seen in controls highlights how endocannabinoid signalling pathways involving the anterior cingulate, hippocampus, and striatum may be disrupted in schizophrenia.

Previous literature has shown that the endogenous $\mathrm{CB}_{1} \mathrm{R}$ agonist, AEA, that is elevated in schizophrenia [66] modulates synaptic plasticity by inhibiting glutamatergic NMDA $\mathrm{Ca} 2+$ flux in the hippocampus, critical for modulating the relative strength and efficacy between synaptic connections [67]. $\mathrm{CB}_{1} \mathrm{R}$ agonists also modulate synaptic plasticity by dysregulating the protein synthesis involved in the formation and degradation of synaptic connections [68]. Our findings extend this work to show that CB1R availability is not associated with glutamate levels in the anterior cingulate. Future work should investigate whether glutamatergic alterations in the striatum and hippocampus are also associated with $\mathrm{CB}_{1} \mathrm{R}$ alterations.

\section{Strengths and limitations}

A strength of the study was that all participants had not been taking any compounds acting on the central nervous system, including pharmacological treatments or illicit substances. While we cannot exclude the possibility that prior use of antipsychotics may have influenced the results, patients had a minimum drug washout period of 6 months and no patients had previously taken depot medications.

Moreover, patients had negative urine drug screens prior to scanning using a test that was able to detect cannabis, cocaine, amphetamine, and opiate use, and we excluded subjects with a history of substance use or dependence.
Prior substance use is, therefore, unlikely to be a significant confound. However, since occasional users may have THCCOOH concentrations below the limit of sensitivity (50 ng/mL) [69], infrequent cannabis use may have been undetected. While some volunteers had previously used cannabis, 1 month of abstinence has been shown to normalise $\mathrm{CB}_{1} \mathrm{R}$ levels [70].

While we cannot exclude the possibility that the correlation between $\mathrm{CB}_{1} \mathrm{R}$ availability and glutamate may have been underpowered, our sample size was comparable to previously published findings investigating the association between dopamine and glutamate in first episode psychosis [27]. While our sample size was powered to detect clinically significant associations $(r>0.4)$, it may have been underpowered to detect smaller associations. However, the clinical significance of smaller associations is unclear. While only males were included due to sex differences in $\mathrm{CB}_{1} \mathrm{R}$ [71], future studies are needed to determine if female patients show $\mathrm{CB}_{1} \mathrm{R}$ alterations and whether these may be linked to glutamatergic function.

Glutamate metabolite estimates acquired using proton magnetic resonance spectroscopy reflect the average signal of intra-cellular and extra-cellular glutamate levels within a particular brain region [72]. In view of the limited spatial resolution of proton magnetic resonance spectroscopy, these findings do not exclude the possibility that pre-synaptic synthesis of glutamate or release may be associated with cannabinoid 1 receptor levels. Previous work has shown that glutamate release can be modulated following the administration of a cannabinoid 1 receptor agonist in rodents [36, 37] and humans [38]. However, we may have failed to observe an association, because we measured the average of both intra-cellular and extra-cellular glutamate. In view of these limitations and that glutamatergic functioning is involved in the dynamic modulation of synaptic connections thought to underlie learning and memory [73, 74], our findings do not exclude the possibility that patients with first episode psychosis may show other alterations in glutamatergic function that are related to CB1 receptors. Although we used a well-validated $1 \mathrm{H}-\mathrm{MRS}$ neuroimaging acquisition proto$\mathrm{col}$, the voxel placement was not rotated to align the anterior cingulate, because GE scanners do not enable voxel rotation handles in their MRS prescription interface.

Future studies using 7-T scanners could be useful as higher field strengths improve the quantification of metabolites and improve signal-to-noise ratios. Previous literature has reported a $2.8 \times$ increase in signal-to-noise ratios and a significant decrease in the variability of metabolite estimates acquired on a 7-Tesla scanner relative to metabolites acquired on a 3-Tesla scanner [75]. These methodological advances will improve the validity and reliability of metabolite estimates and enable us to estimate metabolites present at lower concentrations in the brain $[75,76]$. 


\section{Implications}

The radiotracer which we used, $\left[{ }^{11} \mathrm{C}\right] \mathrm{MePPEP}$, is not displaced by a synthetic analogue of an endogenous cannabinoid, methanandamide [77]. As such, the $\mathrm{V}_{\mathrm{T}}$ of $\left[{ }^{11} \mathrm{C}\right]$ MePPEP is thought to primarily reflect receptor density, not receptor function. Our findings, therefore, indicate that the density of cannabinoid 1 receptor levels has not associated with glutamate levels in the anterior cingulate. However, these findings do not exclude the possibility that the function of the cannabinoid 1 receptor may be altered in patients, and that this may be associated with glutamatergic alterations. The function could be tested in the context of pharmacological challenges using cannabinoid 1 receptor agonists, shown to modulate glutamate levels in rodents [36, 37] and humans [38].

Since $\mathrm{CB}_{1} \mathrm{R}$ binding inhibits calcium entry into the pre-synaptic neuron via N-, P-, and Q-type calcium channels $[32,78]$, fewer $\mathrm{CB}_{1} \mathrm{R}$ may dysregulate calcium and potassium channels, leading to neurochemical alterations in psychosis [27, 79-82]. While we did not show that cannabinoid 1 receptor levels in the anterior cingulate were associated with glutamate levels in the same region, this finding does not exclude the possibility that cannabinoid 1 receptor levels may modulate of neurotransmitters implicated in psychosis including dopamine [83] and gammaaminobutyric acid (GABA) [32]. Future studies are, therefore, needed to investigate if $\mathrm{CB}_{1} \mathrm{R}$ alterations precipitate other neurochemical alterations in psychosis.

\section{Conclusions}

We did not find evidence of a relationship between cannabinoid 1 receptor levels in the anterior cingulate cortex and glutamate levels in the anterior cingulate cortex. However, exploratory analyses indicated that healthy volunteers showed a negative association between ACC glutamate and $\mathrm{CB}_{1} \mathrm{R}$ availability in the striatum and hippocampus; however, these associations were lost in patients. These findings highlight the need for future studies to investigate endocannabinoid signalling pathways, and to investigate whether they may be altered in schizophrenia.

Acknowledgements Dr. Borgan had full access to all the data in the study and take responsibility for the integrity of the data and the accuracy of the data analysis.

Funding F.B. and O.H. were supported by a grant from the EU FP7, METSY. M.V. was supported by grants from the National Institute for Health Research and the Biomedical Research Centre at the South London and Maudsley NHS Foundation trust.

\section{Compliance with ethical standards}

Conflict of interest FB became an employee at COMPASS Pathways Plc after the completion of this work. This work is unrelated to COMPASS Pathways Plc., M.V., and T. M. reported no potential conflicts of interest. O.H. reported receiving investigator-initiated research funding from or participating in advisory or speaker meetings organized by AstraZeneca, Autifony, Bristol-Myers Squibb, Eli Lilly, Heptares, Janssen, Lundbeck, Lyden-Delta, Otsuka, Servier, Sunovion, RAND, and Roche. J.H. reported speaker fees in meetings organized by Orion Pharma, Lundbeck, and Otsuka.

Open Access This article is licensed under a Creative Commons Attribution 4.0 International License, which permits use, sharing, adaptation, distribution and reproduction in any medium or format, as long as you give appropriate credit to the original author(s) and the source, provide a link to the Creative Commons licence, and indicate if changes were made. The images or other third party material in this article are included in the article's Creative Commons licence, unless indicated otherwise in a credit line to the material. If material is not included in the article's Creative Commons licence and your intended use is not permitted by statutory regulation or exceeds the permitted use, you will need to obtain permission directly from the copyright holder. To view a copy of this licence, visit http://creativecommons.org/licenses/by/4.0/.

\section{References}

1. Ustün TB et al (1999) Multiple-informant ranking of the disabling effects of different health conditions in 14 countries. WHO/NIH joint project CAR study group. Lancet (Lond Engl) 354:111-115

2. Chong HY et al (2016) Global economic burden of schizophrenia: a systematic review. Neuropsychiatr Dis Treat 12:357-373

3. Ayesa-Arriola R et al (2013) Long-term (3-year) neurocognitive effectiveness of antipsychotic medications in first-episode nonaffective psychosis: a randomized comparison of haloperidol, olanzapine, and risperidone. Psychopharmacology 227:615-625

4. Meltzer HY (1997) Treatment-resistant schizophrenia: the role of clozapine. Curr Med Res Opin 14:1-20

5. Kaar SJ, Natesan S, McCutcheon R, Howes OD (2019) Antipsychotics: mechanisms underlying clinical response and sideeffects and novel treatment approaches based on pathophysiology. Neuropharmacology. https://doi.org/10.1016/j.neuropharm .2019 .107704

6. Friedman JI et al (2002) A double blind placebo controlled trial of donepezil adjunctive treatment to risperidone for the cognitive impairment of schizophrenia. Biol Psychiatry 51:349-357

7. Friedman JI et al (2008) A pilot study of adjunctive atomoxetine treatment to second-generation antipsychotics for cognitive impairment in schizophrenia. J Clin Psychopharmacol 28:59-63

8. Borgan F et al (2019) The neural and molecular basis of working memory function in psychosis: a multimodal PET-fMRI study. Mol Psychiatry. https://doi.org/10.1038/s41380-019-0619-6

9. McCutcheon RA, Reis Marques T, Howes OD (2020) Schizophrenia: an Overview. JAMA Psychiatry 77:201-210

10. Borgan F et al (2019) The effects of cannabinoid 1 receptor compounds on memory: a meta-analysis and systematic review across species. Psychopharmacology 236:3257-3270

11. Howes O, McCutcheon R, Stone J (2015) Glutamate and dopamine in schizophrenia: an update for the 21 st century. J Psychopharmacol 29:97-115 
12. Javitt DC (2007) Glutamate and schizophrenia: phencyclidine, $\mathrm{N}$-methyl-D-aspartate receptors, and dopamine-glutamate interactions. Int Rev Neurobiol 78:69-108

13. Javitt DC, Zukin SR (1991) Recent advances in the phencyclidine model of schizophrenia. Am J Psychiatry. 148(10):1301-8. https ://doi.org/10.1176/ajp.148.10.1301

14. Olney JW, Farber NB (1995) Glutamate receptor dysfunction and schizophrenia. Arch Gen Psychiatry 52:998-1007

15. Olney JW, Newcomer JW, Farber NB (1999) NMDA receptor hypofunction model of schizophrenia. J Psychiatr Res 33:523-533

16. Stone JM et al (2012) Ketamine effects on brain GABA and glutamate levels with $1 \mathrm{H}-\mathrm{MRS}$ : relationship to ketamine-induced psychopathology. Mol Psychiatry 17:664-665

17. Taylor MJ, Tiangga ER, Mhuircheartaigh RN, Cowen PJ (2012) Lack of effect of ketamine on cortical glutamate and glutamine in healthy volunteers: a proton magnetic resonance spectroscopy study. J Psychopharmacol 26:733-737

18. Bojesen KB et al (2018) Glutamate levels and resting cerebral blood flow in anterior cingulate cortex are associated at rest and immediately following infusion of S-ketamine in healthy volunteers. Front psychiatry 9:22

19. Bustillo JR et al (2011) Glutamate as a marker of cognitive function in schizophrenia: a proton spectroscopic imaging study at 4 tesla. Biol Psychiatry 69:19-27

20. Kumar J et al (2018) Glutathione and glutamate in schizophrenia: a 7T MRS study. Psychiatry Mol. https://doi.org/10.1038/s4138 0-018-0104-7

21. Théberge $\mathbf{J}$ et al (2002) Glutamate and glutamine measured with 4.0 T proton MRS in never-treated patients with schizophrenia and healthy volunteers. Am J Psychiatry 159:1944-1946

22. Bartha $\mathrm{R}$ et al (1997) Measurement of glutamate and glutamine in the medial prefrontal cortex of never-treated schizophrenic patients and healthy controls by proton magnetic resonance spectroscopy. Arch Gen Psychiatry 54:959-965

23. Egerton A et al (2018) Response to initial antipsychotic treatment in first episode psychosis is related to anterior cingulate glutamate levels: a multicentre 1H-MRS study (OPTiMiSE). Mol Psychiatry. https://doi.org/10.1038/s41380-018-0082-9

24. Chen $\mathrm{T}$ et al (2017) Abnormal concentration of GABA and glutamate in the prefrontal cortex in schizophrenia.-An in vivo 1H-MRS study. Shanghai Arch Psychiatry 29:277-286

25. Borgan FR et al (2019) Glutamate levels in the anterior cingulate cortex in un-medicated first episode psychosis: a proton magnetic resonance spectroscopy study. Sci Rep 9:8685

26. Wang $J$ et al (2016) Reduced $\gamma$-aminobutyric acid and glutamate+glutamine levels in drug-naïve patients with firstepisode schizophrenia but not in those at ultrahigh risk. Neural Plast 2016:1-9

27. Jauhar S, McCutcheon R, Borgan F, Veronese M, Nour M, Pepper F, Rogdaki M, Stone J, Egerton A, Turkheimer F, McGuire P, Howes O (2018) The relationship between cortical glutamate and striatal dopamine function in first episode psychosis: a multi-modal PET and MRS imaging study. Lancet Psychiatr 5(10):816-823

28. Herkenham L, Little J, Melvin, De Costa R (1990) Cannabinoid receptor localization in brain. Proc Natl Acad Sci 87:1932-1936

29. Wilson RI, Nicoll RA (2001) Endogenous cannabinoids mediate retrograde signalling at hippocampal synapses Marijuana affects brain function primarily by activating the G- protein-coupled cannabinoid receptor-1 (CB1). Lett to Nat 401:588-592

30. Wang H, Lupica CR (2014) Release of endogenous cannabinoids from ventral tegmental area dopamine neurons and the modulation of synaptic processes. Prog Neuropsychopharmacol Biol Psychiatry 52:24-27

31. Pertwee RG (2008) The diverse $\mathrm{CB}_{1}$ and $\mathrm{CB}_{2}$ receptor pharmacology of three plant cannabinoids: $\Delta^{9}$-tetrahydrocannabinol, cannabidiol and $\Delta{ }^{9}$-tetrahydrocannabivarin. Br J Pharmacol 153:199-215

32. Wilson RI, Kunos G, Nicoll RA (2001) Presynaptic specificity of endocannabinoid signaling in the hippocampus. Neuron 31:453-462

33. Kano M, Ohno-Shosaku T, Hashimotodani Y, Uchigashima M, Watanabe M (2009) Endocannabinoid-mediated control of synaptic transmission. Physiol Rev 89:309-380

34. Khani A et al (2015) Activation of cannabinoid system in anterior cingulate cortex and orbitofrontal cortex modulates cost-benefit decision making. Psychopharmacology 232:2097-2112

35. Guo B et al (2018) Chronic inflammatory pain impairs mglur5mediated depolarization-induced suppression of excitation in the anterior cingulate cortex. Cereb Cortex 28:2118-2130

36. Ferraro L et al (2001) The cannabinoid receptor agonist WIN 55,212-2 regulates glutamate transmission in rat cerebral cortex: an in vivo and in vitro study. Cereb Cortex 11:728-733

37. Gerdeman G, Lovinger DM (2001) CB1 cannabinoid receptor inhibits synaptic release of glutamate in rat dorsolateral striatum. J Neurophysiol 85:468-471

38. Colizzi M et al (2019) Delta-9-tetrahydrocannabinol increases striatal glutamate levels in healthy individuals: implications for psychosis. Mol Psychiatry. https://doi.org/10.1038/s4138 0-019-0374-8

39. Monory K et al (2006) The endocannabinoid system controls key epileptogenic circuits in the hippocampus. Neuron 51:455-466

40. Marsicano G, Goodenough S, Monory K, Hermann H, Eder M, Cannich A et al (2003) CB1 cannabinoid receptors and on-demand defense against excitotoxicity. Science 302(5642):84-8

41. Borgan F et al (2019) In vivo availability of cannabinoid 1 receptor levels in patients with first-episode psychosis. JAMA Psychiatry. https://doi.org/10.1001/jamapsychiatry.2019.1427

42. Borgan F, O'Daly O, Huang K, Veronese M, Withers D, Batterham R, Howes O (2019) Neural responsivity to food cues in un-medicated first episode psychosis: an fMRI study. JAMA Netw Open 2(1):e186893

43. Ranganathan $\mathrm{M}$ et al (2016) Reduced brain cannabinoid receptor availability in schizophrenia. Biol Psychiatry 79:997-1005

44. Wechsler D (1997) Wechsler adult intelligence scale, 3rd edn. The psychological corporation, San Antonio

45. Gonzalez-Blanch $\mathrm{C}$ et al (2011) A digit symbol coding task as a screening instrument for cognitive impairment in first-episode psychosis. Arch Clin Neuropsychol 26:48-58

46. First M, Spitzer R, Miriam G, Williams J (2007) Structured clinical interview of DSM-IV-TR AXIS I disoderss-patient edition (SCID-I/P, 1/2007 revision) (Biometrics research)

47. Maxwell E (1992) Family interview for genetic studies (FIGS): a manual for FIGS: clinical neurogenetics branch. Intramural research program, National institute of mental health

48. Kay SR, Fiszbein A, Opler LA (1987) The positive and negative syndrome scale (PANSS) for schizophrenia. Schizophr Bull $13: 261-276$

49. Aas IH (2011) Guidelines for rating Global Assessment of Functioning (GAF). Ann Gen psychiatr 10(1):2. https://doi. org/10.1186/1744-859X-10-2

50. Yasuno $\mathrm{F}$ et al (2008) The PET radioligand [11C]MePPEP binds reversibly and with high specific signal to cannabinoid CB1 receptors in nonhuman primate brain. Neuropsychopharmacology 33:259-269

51. Riaño Barros DA et al (2014) Test-retest reproducibility of cannabinoid-receptor type 1 availability quantified with the PET ligand $\left[{ }^{11} \mathrm{C}\right]$ MePPEP. Neuroimage 97:151-162

52. Borgan F et al (2019) Neural Responsivity to Food Cues in Patients With Unmedicated First-Episode Psychosis. JAMA Netw Open 2:e186893 
53. The Mathworks, I. MATLAB. (2010)

54. Montgomery AJ et al (2006) Correction of head movement on PET studies: comparison of methods. J Nucl Med 47:1936-1944

55. Hammers A et al (2003) Three-dimensional maximum probability atlas of the human brain, with particular reference to the temporal lobe. Hum Brain Mapp 19:224-247

56. Feng D, Huang SC, Wang XM (1993) Models for computer-simulation studies of input functions for tracer kinetic modeling with positron emission tomography. Int J Biomed Comput 32:95-110

57. Tonietto $\mathrm{M}$ et al (2018) A unified framework for plasma data modelling in dynamic positron emission tomography studies. IEEE Trans Biomed Eng 9294:1-1

58. Egerton A et al (2012) Anterior cingulate glutamate levels related to clinical status following treatment in first-episode schizophrenia. Neuropsychopharmacology 37:2515-2521

59. Brugger SP, Howes OD (2017) Heterogeneity and homogeneity of regional brain structure in schizophrenia. JAMA Psychiatry 74:1104

60. Fortin DA, Levine ES (2007) Differential effects of endocannabinoids on glutamatergic and GABAergic inputs to layer 5 pyramidal neurons. Cereb Cortex 17:163-174

61. Gasparovic C, Chen H, Mullins PG (2018) Errors in ${ }^{1}$ H-MRS estimates of brain metabolite concentrations caused by failing to take into account tissue-specific signal relaxation. NMR Biomed 31:e3914

62. IBM, C. Statistical parametric mapping software. IBM SPSS statistics for windows, version 22 (2013)

63. Lehoux C, Gobeil MH, Lefèbvre AA, Maziade M, Roy MA (2009) The five-factor structure of the PANSS: a critical review of its consistency across studies. Clin Schizophr Relat Psychoses 3:103-110

64. Németh B, Ledent C, Freund TF, Hájos N. CB 1 receptor-dependent and -independent inhibition of excitatory postsynaptic currents in the hippocampus by WIN 55,212-2. https://doi.org/10.1016/j. neuropharm.2007.07.003

65. Fortin DA, Levine ES (2006) Differential Effects of Endocannabinoids on Glutamatergic and GABAergic Inputs to Layer 5 Pyramidal Neurons. Cereb Cortex 17:163-174

66. Minichino A et al (2019) Measuring disturbance of the endocannabinoid system in psychosis: a systematic review and metaanalysis. JAMA Psychiatry 76:914-923

67. Hampson AJ et al. Dual effects of anandamide on nmda receptormediated responses and neurotransmission. J. Neurochem. Lippincott-Raven Publ

68. Navakkode S, Korte M (2014) Pharmacological activation of CB1 receptor modulates long term potentiation by interfering with protein synthesis. Neuropharmacology 79:525-533

69. Musshoff F, Madea B (2006) Review of biologic matrices (urine, blood, hair) as indicators of recent or ongoing cannabis use. Ther Drug Monit 28:155-163
70. D'Souza DC et al (2016) Rapid changes in CB1 receptor availability in cannabis dependent males after abstinence from cannabis. Biol Psychiatry Cogn Neurosci Neuroimag 1:60-67

71. Laurikainen $\mathrm{H}$ et al (2018) Sex difference in brain CB1 receptor availability in man. Neuroimage. https://doi.org/10.1016/j.neuro image.2018.10.013

72. Poels EMP et al (2014) Glutamatergic abnormalities in schizophrenia: a review of proton MRS findings. Schizophr Res 152:325-33212

73. Baez MV, Cercato MC, Jerusalinsky DA (2018) NMDA receptor subunits change after synaptic plasticity induction and learning and memory acquisition. Neural Plast 2018:1-11

74. Malenka RC (1995) Review $\mathbf{\square}$ : LTP and LTD: dynamic and interactive processes of synaptic plasticity. Neurosci 1:35-42

75. Rodgers CT et al (2014) Human cardiac ${ }^{31} \mathrm{P}$ magnetic resonance spectroscopy at 7 tesla. Magn Reson Med 72:304-315

76. Otazo R, Mueller B, Ugurbil K, Wald L, Posse S (2006) Signalto-noise ratio and spectral linewidth improvements between 1.5 and 7 Tesla in proton echo-planar spectroscopic imaging. Magn Reson Med 56:1200-1210

77. Terry GE et al (2010) Imaging and quantitation of cannabinoid CB1 receptors in human and monkey brains using (18)F-labeled inverse agonist radioligands. J Nucl Med 51:112-120

78. Szabo GG et al (2014) Presynaptic calcium channel inhibition underlies CB1 cannabinoid receptor-mediated suppression of gaba release. J Neurosci 34:7958-7963

79. Merritt K, Egerton A, Kempton MJ, Taylor MJ, McGuire PK (2016) Nature of Glutamate Alterations in Schizophrenia. JAMA Psychiatry 73:665

80. McCutcheon R, Beck K, Jauhar S, Howes OD (2017) Defining the locus of dopaminergic dysfunction in schizophrenia: a metaanalysis and test of the mesolimbic hypothesis. Schizophr Bull. https://doi.org/10.1093/schbul/sbx180

81. Watis L, Chen SH, Chua HC, Chong SA, Sim K (2008) Glutamatergic abnormalities of the thalamus in schizophrenia: a systematic review. J Neural Transm 115:493-511

82. Kraguljac NV, White DM, Reid MA, Lahti AC (2013) Increased hippocampal glutamate and volumetric deficits in unmedicated patients with schizophrenia. JAMA Psychiatry 70:1294

83. Melis M et al (2004) Cellular/molecular endocannabinoids mediate presynaptic inhibition of glutamatergic transmission in rat ventral tegmental area dopamine neurons through activation of CB1 receptors. J Nuerosci. https://doi.org/10.1523/JNEUR OSCI.4503-03.2004

84. Coodin S (2001) Body mass index in persons with schizophrenia. Can J Psychiatry 46:549-555 